\title{
Exposure of pumping intense external electromagnetic fields on radioelectronic engineering substances with domain structure
}

\author{
Larissa Cherckesova ${ }^{1, *}$, and Dmitriy Bezuglov ${ }^{2}$ \\ ${ }^{1}$ Don State Technical University, 344000 Rostov-on-Don Gagarina 1, Russia \\ ${ }^{2}$ Rostov Branch of Russian Customs Academy, 344002 Rostov-on-Don Budennovskiy 20, Russia
}

\begin{abstract}
Recently, the research of interaction processes of materials with domain structure, applied in radioelectronic techniques, with strong electromagnetic fields of external influence - pumping represents the special interest. Similar processes underlies on the ground of many electronic devices functioning - of quantum, optical, etc. It leads to change of properties and constructions of radioelectronic techniques with domain structure materials, to define of their elementary power processes, phase transitions. Investigations were carried out by means of general interaction processes mathematical model of the strong (intense) field of pumping external influence with radioelectronic techniques materials elements, in which the magnetic, polar, dielectric, optical, elastic and other properties are developing.
\end{abstract}

\section{Introduction}

At present, the investigation of interaction processes of substances with domain structure, applied in radioelectronic engineering and techniques, with intense (strong) electromagnetic fields of external influence of pumping represents the special interest.

For a long time is positioned, that at affecting of intense strong external fields, in the substances and materials with domain structure of radio- electronics the anomal phenomena, effects and processes, result to modification of their composition and structure, which while are insufficiently studied are displayed $[1,2]$.

\section{Mathematical modeling}

The element of substance or material of radioelectronics is routinely characterized by the response (state coordinate) $y$ on the external influence (pumping) $x$, and steepness of characteristics (transfer function or conversion coefficient) $S$ [3].

The dependence $y=S x$ is constitutive equation (or transformation equation). This dependence can be linear, nonlinear, single-valued or multi-valued (hysteresis loops).

Steepness $S$ is determined by chemical composition, structure and an internal energy of substance or material of radioelectronic engineering [4].

\footnotetext{
* Corresponding author: chia2002@inbox.ru
} 
On Fig. 1 the diagram of hysteresis loop and its derivatives is presented.

The dashed line results the family of symmetrical hysteresis loops, received at various values of maximal field of pumping $x_{\max }$ (Fig. 1,a).

The cycles of dynamic steepness (or differential conversion coefficient), $S_{D}=y_{x}^{\prime}$, corresponding to the family of symmetrical hysteresis loops, are shown on Fig. 1, b. $\gamma_{4}$.

On Fig. $1, c$ is shown the curvature of characteristics $S_{D}^{\prime}=y_{x x}^{\prime \prime}$ for the limiting hysteresis loop, representing the first derivative of dynamic steepness $S_{D}$.

At modification of the maximal field of pumping $x_{\max }$ the corresponding points of the response $y_{\max }$ move, at first, by the basic curve of transformation $(B C T)$ (fig. $1, d$, the solid curve), and then - on the limiting hysteresis loop [5].

In the capacity of $B C T$ can figure the magnetisation curves, polarisation curves, elasticity curves, inertia (sluggishness) curves, etc. To the limiting hysteresis loop corresponds the boundary field of pumping $x^{\text {bnd }}$; thus, the researching substance or material of radioelectronic engineering is near (is close) to saturation [6].

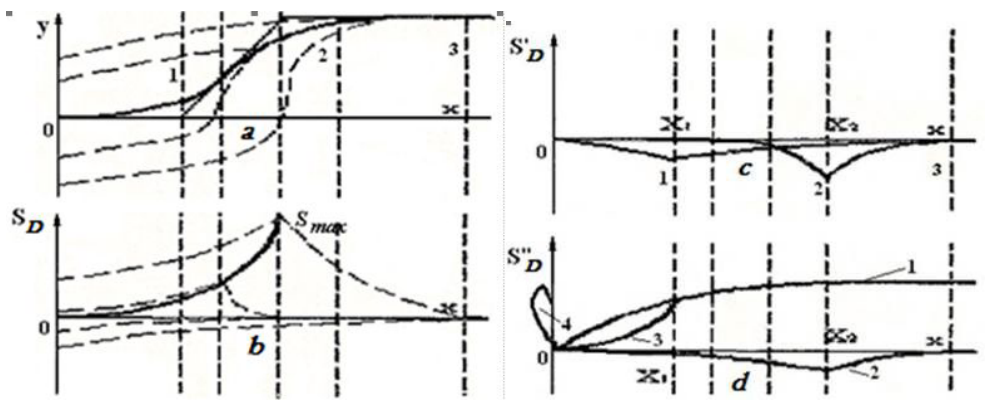

Fig. 1. Diagram of hysteresis loop and its derivatives.

The basic physical values, characterizing the hysteresis loop, are: the saturation induction $y_{s}$, the residual (remanent) induction $y_{r}$, the coercive force $H_{c}$, the initial (starting) steepness of characteristics $S_{\text {init }}$, the maximal steepness of characteristics $S_{\text {max }}$, and the value $S_{\max }$ can be received at $x=H_{c}$.[7].

\section{Analysis of interacting mechanism}

By analytical consideration of the interacting mechanism of substance or material of radioelectronic engineering having domain structure, with the field of external influence of pumping, it is necessary to define the characteristic points, the quantity of positions (locations) of equilibrium (balance) points and their characteristics (stable or unstable), the amount and order (sort) of transitions of the substance or the material from one state to another, during the period [8].

There is set of analytical approximations of the basic curve of transformation $(B C T)$. We'll take advantage of piecewise-linear approximation. Thus, $B C T$ is approximated by three segments (Fig. 1,a). The type of pumping regime (mode) is marked out by index, corresponding to consecutive states of substance or material in the time.

The regime on the segment $0-1$ corresponds to weak field of pumping $x<x_{\max }$ at which in the substance of material happen the elastic (reversible) processes. At sinusoidal external influence in the segment $0-1$ at weak field of pumping, the response of the 
substance or material will be also sinusoidal, i.e. the linear regime (condition) is observed. On the segment $1-2$ there are inelastic (irreversible) processes. To the segment $2-3$ there is corresponding the elastic reversible process again [9].

On the other hand, all analytical continuous approximations of the basic curve of transformation $(B C T)$ and their derivatives do not consider an initial (starting) segment. For example, for approximation of the function $x=\alpha \operatorname{sh} \beta$, where $\alpha$ and $\beta$ are constant coefficients, (Fig. 1, $d$, curve 1), it is possible to determine the coordinates of point of upper «knee» (Fig. 1, d, curve 2) and it is difficultly to define the coordinates of lower «knee».

However, as the investigations have shown, equilibrium (balance) points 1 and 2 play the fundamental role at the determination and research of mechanism of interacting of substance and materials of radioelectronic engineering and techniques with intensive external influence - high power field of pumping. Therefore at analytical calculations appear the necessity of «linkage» of analytical continuous approximations of $B C T$ and its continuous derivatives. Considering, that such approximations are nonlinear functions, is obviously, that such approach calls the great difficulties even for simple tasks. This contradiction calls the rupture (break) between possibilities of the theory of nonlinearities calculation and the experimental practice [10].

\section{Method of solution}

For the solvability of this problem the following method is offered. We will take advantage of steepness cycles (Fig. 1, b). At amplitude change of pumping field $x_{\max }$ the corresponding point of steepness $S$ moves through maximal values $S_{\max }$ (Fig. 1, solid curve of the symmetrical steepness cycles). They correspond to the family of symmetrical steepness cycles - the basic curvature of curve $(B C C)$. The introduced thus characteristics $\mathrm{BCC}$ is easy for receiving experimentally, having fulfilled the measurings by means of voltammeter, and having differentiated the corresponding values of the basic curve of transformation (BCT). The carried-out analysis of the basic curvature of curve $(B C C)$, experimentally received for various substances and materials of radioelectronic techniques (magnetic and polar), has shown, that they are convenient for approximation of crossly inverse functions of the basic curve of transformation (BCT) [2].

Exploring on extremal values the function $x_{S}=\alpha \operatorname{sh} \beta$, first $x_{S}^{\prime}$ and second $x_{S}^{\prime \prime}$ derivatives, we'll find on extremumes the coordinates $s_{1}$ and $x_{1}$, and on $x_{1}$ it is possible to find the values $y_{1}$, i.e. coordinates of equilibrium (balance) of the point of lower «knee». On Fig. 1, $d$ the graphs of functions $S_{D}$ and $x_{S}^{\prime \prime}$ (curves 3 and 4 accordingly) are resulted.

Let's observe the behaviour of substance or material with domain structure at certain temperature as the system of harmonious and anharmonic oscillations of atoms and molecules. Potential (elastic) energy $V_{n}$ of microparticles depends both on elastic forces of connection, and from temperature, and is determined by the equation [10]:

$$
V_{n}=0,5 k y^{2}+0,25 k_{a} y^{4}
$$

where $y$ - displacement (coordinate), $k$ and $k_{a}$ - constant coefficients of elasticity and anharmonicity. Values of coefficients $k$ and $k_{a}$ depend on temperature and pressure, coefficient $k_{a}$ is always positive, and the coefficient $k$ can take over and negative values.

Let's analyze the dynamics of processes in the substance or material with domain structure (Fig. 2). The stable position (location) of microparticles is determined by the minimum of potential energy $V_{n}$. Equating the derivative $V_{n y}^{\prime}$ to null, we'll receive, that for 
$k>0$ the minimum $V_{n}$ corresponds to the point $y=0$, and for $k<0$ - to points $y= \pm \sqrt{|k|\left(k_{a}\right)^{-1}}$ (Fig. 2, a, curves 1 and 2 accordingly).

At presence of domain (spontaneous) polarisation $P_{s}$ or magnetization $J_{s}$ in the expression for $V_{n}$ it is necessary to take into account the potential energy of particles, called by appearance of internal field. Then centers of equilibrium (balance) can displace.

Domain (spontaneous) polarization - magnetization is possible, when the value of forces of internal field will exceed the value of elastic forces [6], [7].
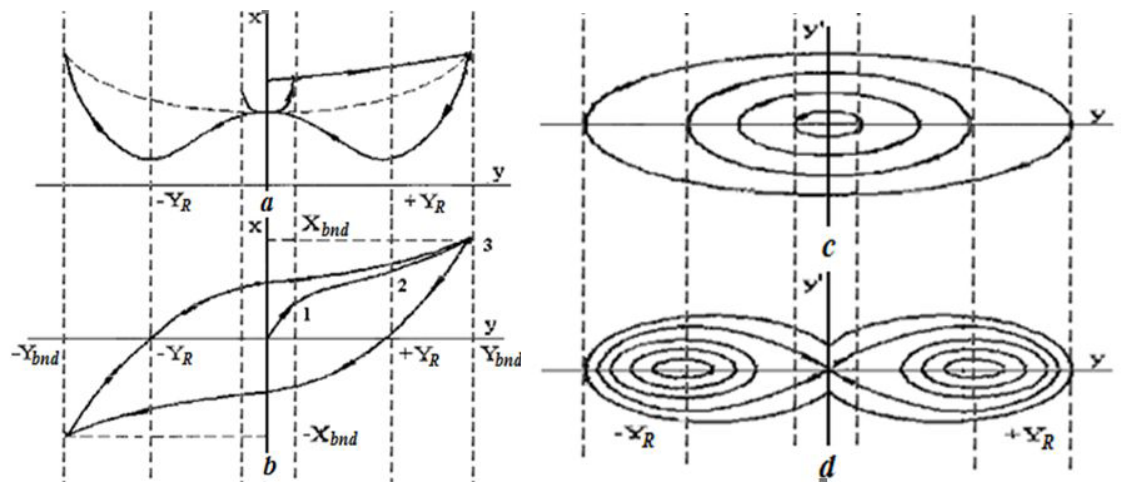

Fig. 2. Dynamics of processes in the substances and materials with domain structure.

Let's suppose that originally in the substance of material with field of pumping $x$ the values $P_{s}$ or $J_{s}$ are equal to null. We'll add to substance of material of radioelectronic engineering and technique the weak field of pumping $x_{1}$, then the connection between $y$ and $x$ is close to linear, thus the potential energy increase to value $V_{1}$ (Fig. 2, $b$, segment 1-2).

The further increase of pumping to $x_{b n d}$ result to reorientations of all moments of magnetic domains, incoincident with direction of the field of pumping, up to saturation. Thus the value $V_{n}$ increases to values $y_{b n d}$. At decrease of the field of pumping to null $x=0$ the response changes under the descending curve from $y_{b n d}$, to $y_{R}^{+}$, the residual polarization $P_{R}$ or magnetisation $J_{R}$ is observed, and the part of domains remains oriented in the positive direction. This state corresponds to the minimum of potential energy and will be stable.

The further increasing $x$ in the negative direction to $x=-x_{c}$ result to reorientations of all domains in this direction and there ensues the equilibrium (balance) state, when the moments of domains with different signs are equal to each other. Thus, the potential energy will be maximal that will correspond to unstable state [10].

Increasing of the field of pumping to $-x_{b n d}$ in the negative direction result to reorientations of all domains in this direction up to saturation. Thus the potential energy changes on the dotted curve (Fig. 2, a), that corresponds to descending branch of hysteresis loop from $-x_{c}$ to $-x_{\text {bnd }}$.

Behaviour of the moments of particles domains in the substance of materials of radioelectronic engineering and techniques, depending on the form of potential energy, is visually demonstrated by phase portraits (trajectories) [6], [7].

Let the particles (moments of particles domains) accomplish the harmonic oscillations in the form $y=y_{\max } \sin \omega t$ with velocity $y^{\prime}=\omega y_{\max } \cos \omega t$, then the motion on the phase 
plane happens near (around) the stable location of centre, corresponding to $y=0$ and to minimum of potential energy.

Thus phase trajectories are represented by ellipse curves. The field of these oscillations is limited by two separatrixes, which transit through singular point of «saddle» type at $y=y^{\prime}=0$, phase trajectories are out of the field, limited by separatrixes, correspond to the motions of overturning, i.e. particle transition (reorientation of domains moments) from one state in another.

Let's ascertain a role of phenomena of the anomal modulation of power - consuming parameter in the mechanism of interacting of substance or material with intensive pumping, that is in regime (condition), close to saturation. On Fig. 3 the dependences of dynamic steepness of substance or material characteristics of $S_{\tau}$ (Fig. 3, a), it's first $S_{\tau}^{\prime}$ (Fig. 3, b) and second $S_{\tau}^{\prime \prime}$ (Fig. 3,c) derivatives, computed with the account of harmonious field of pumping $x=x_{\max } \omega t$ at $x_{\max }=0,5 ; 1,0 ; 4,0 ; 10,0$ are resulted (curves $1,2,3,4$ ) on initial equation:

$$
S_{\tau}=\frac{S_{0}}{\operatorname{ch}\left(\beta \cdot x_{\max } \sin \omega t\right)}
$$

where $S_{0}$ - statistical steepness.

From resulted on Fig. 3 curves it is visible, that with growth of pumping amplitude $x_{\max }$ the depth (intensity) of parameter modulation of dynamic steepness is increased, and the square limited by dynamic steepness curve and time axis (i.e. integral steepness) decreases:

$$
S_{I}=\int_{0}^{T} S(t) d t,
$$

where $T$ - period of steepness modulation.

At excess by amplitude of value $x_{\max }>0,5$ is observed the nonlinear abnormal change of parameter of integral steepness $S_{I}$ (curves 2, 3, 4), with growth $x_{\max }$ proportionally to change $S_{n}$. Thus the depth of modulation (curves 3,4 ) loses the physical sense.

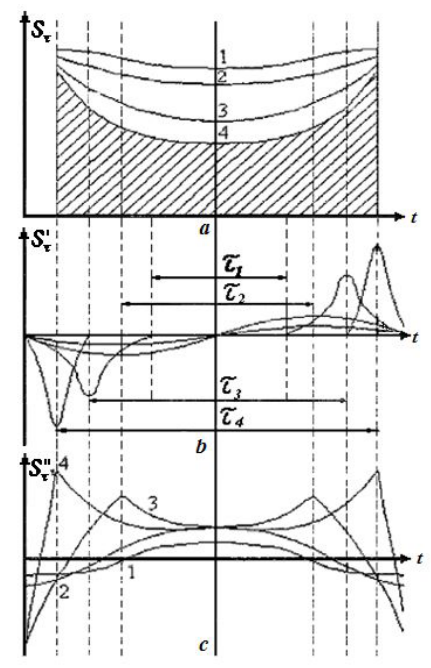

Fig 3. Dependencies of dynamic steepness of substance or material characteristics $S_{\tau}$ (a), its first $S_{\tau}^{\prime}(\mathrm{b})$ and second $S_{\tau}^{\prime \prime}(\mathrm{c})$ derivatives. 
At study of the mechanism of power interchange in controllabled reactive elements the conceptions use: the maximal and minimal parameters. In this case $S_{\max }=S_{0} T$, and $S_{\min }=S_{I}$, i.e. the minimal value of the characteristics steepness is equal to its integral steepness at corresponding field of pumping of high (intensive) power.

Then the estimation of physical processes and effect degree of external influence can be realized by means of the transformation effectiveness coefficient (integral coefficient of modulation depth) $m_{z}$, representing the relationship of peak-to-peak double amplitude of integral parameter change $\Delta S=S_{0} T-S_{I}$ to maximal value of integral parameter during period of it change $[6,7]$ :

$$
m_{z}=\left(1-S_{I}\right) /\left(S_{0} T\right) ; \text { or } m_{z}=\left(1-S_{I}^{\text {aver }}\right) /\left(S_{0}\right) ; S_{I}^{\text {aver }}=S_{I} / T
$$

Phenomena of integral modulation of dynamic parameter of element consists that at excess of external influence the boundary value of linear regime (condition) the parameter modulation depth is aimed to limiting value. The offered method of integral modulation of dynamic parameters of substances and materials with domain structure, using in radioelectronic engineering and techniques (at the before given temperature) can be applied at any intensity of the fields of pumping - weak, average, intensive (strong).

\section{Experimental results}

Analogous to Fig. 3, c, the dynamic curves are received for semiconducting and segnetoelectric (ferroelectric) substances experimentally, on the oscillograph screen.

Depending on external influence value they are resulted on Fig. 4, where cases a - d correspond to curves $1-4$ on Fig. 3, c. It proves the theoretical leading-outs made above.

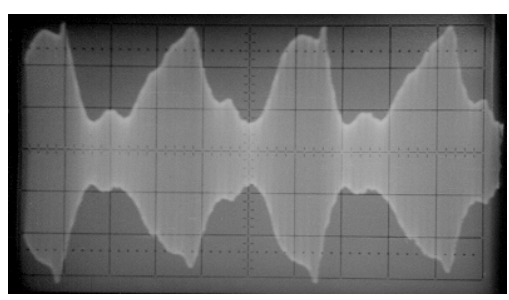

(a)

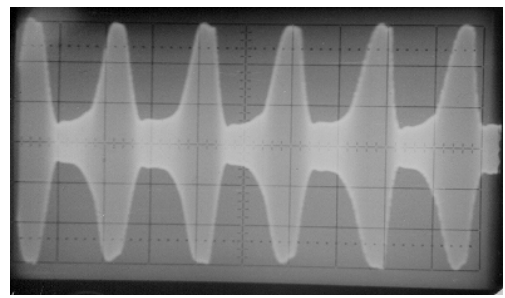

(b)

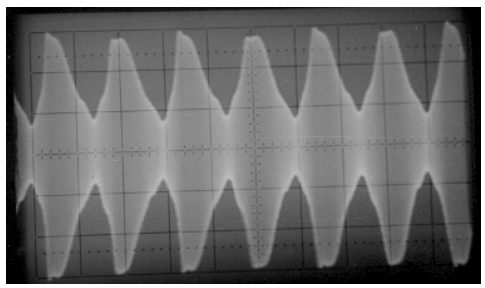

(b)

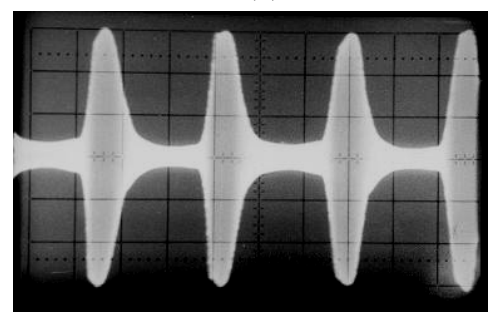

(d)

Fig. 4. Dynamic curves received experimentally for semiconducting and segnetoelectric (ferroelectric) substances and materials. 


\section{Conclusions}

Thus, in this article the processes happening at interacting of substances and materials of radioelectronic engineering and techniques with domain structure, with the intensive (strong) electromagnetic fields of external influences - pumping is explored. It is ascertained, that the phenomenon of integral modulation of element dynamic parameter consists that at excess of external influence of boundary value of linear regime the depth of parameter modulation is aimed to the limiting value. Thus, the integral parameter decreases and the energy of different - polar pulses is increased proportionally to intensity of external influence; there is happening the disintegration of the pulses phases, also proportional to velocity of parameter change; the interaction time decreases and the time interval between extremums of the parameter change velocity is increased. The offered method of integral modulation of dynamic parameters of substances and materials with domain structure, applied in radioelectronic engineering and techniques (at the given temperature) can be applied at pumping fields external influence of any intensity - weak, average, intensive (strong).

This work was supported by Russian Ministry of Education and Science in accordance to the Government Decree № 218 from April 9, 2010 (project number № 074-11-2018-013 from May 31, 2018 (03.G25.31.0284)).

\section{References}

1. D.A. Bezuglov, L.V. Cherckesova, N.N. Prokopenko, G.P. Sinyavsky, G.N. Shalamov, 2015 IEEE COMCAS, 1 (2015) doi: 10.1109/COMCAS.2015.7360435

2. D.A. Bezuglov, L.V. Cherckesova, N.N. Prokopenko, G.P. Sinyavsky, G.N. Shalamov, 2015 IEEE COMCAS, 1 (2015) doi: 10.1109/COMCAS.2015.7360434

3. D.A. Bezuglov, G.P. Sinyavsky, L.V. Cherckesova, G.N. Shalamov and A.N. Zaichenko, ICATT, 351 (2015) doi: 10.1109/ICATT.2015.7136881

4. D.A. Bezuglov, G.P. Sinyavsky, L.V. Cherckesova, O.N. Manaenkova, 2015 International Conference on Antenna Theory and Techniques (ICATT), 363 (2015)

5. D.A. Bezuglov, G.P. Sinyavsky, L.V. Cherckesova, G.N. Shalamov, A.N. Zaichenko, International Conference on Antenna Theory and Techniques (ICATT), 360 (2015)

6. D.A. Bezuglov, G.P. Sinyavsky, L.V. Cherckesova, G.N. Shalamov, A.N. Zaichenko, SIBCON, 1 (2015) doi: 10.1109/SIBCON.2015.7147269

7. D.A. Bezuglov, G.P. Sinyavsky, L.V. Cherckesova, G.N. Shalamov, A.N. Zaichenko, Intern. Siberian Conference on Control and Communications (SIBCON), 1 (2015)

8. D.A.Bezuglov, G.P. Sinyavsky, L.V. Cherckesova, O.N. Manaenkova, SIBCON'2015, IEEE, 1 (2015)

9. D.A. Bezuglov, G.P. Sinyavsky, L.V. Cherckesova, G.N. Shalamov, A.G. Shein, O.N. Manaenkova, A.N. Zaichenko, IEEE East-West Design \& Test Symposium (EWDTS), 1 (2015) doi: 10.1109/EWDTS.2015.7493171

10. D.A. Bezuglov, M.Yu. Zvezdina, L.V. Cherckesova, Yu.A. Shokova, N.N. Prokopenko, G.N. Shalamov, G.P. Sinyavsky, O.N. Manaenkova IEEE East-West Design \& Test Symposium (EWDTS), 1 (2016) 